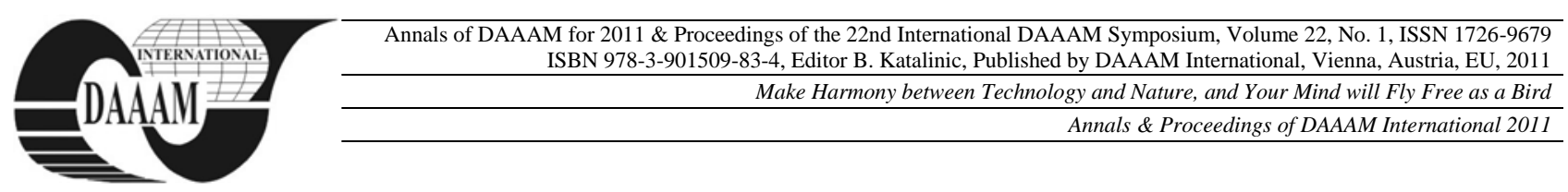

\title{
MECHANISMS OF ENERGY ABSORPTION AND YIELDING OF COMPOSITE STRUCTURES
}

\author{
DUMITRACHE, R[amona]; DUMITRACHE, C[osmin] L[aurentiu] \& CHIRCOR, M[ihael]
}

\begin{abstract}
The cracks can propagate in any direction and in any plan in relation to the main stress direction, according to the local tensions as well as to the material's features and some of these cracks can damage the material's behavior by decreasing its strength. Broken fibers decrease the traction strength, while the delaminations among adjacent layers decrease the compression strength. An effective method to improve the impact features of graphite fibers reinforced composite materials is to add a small percentage of fibers with high strength, this leading to higher performances under impact. Key words: metallic materials, fiber, impact, energy, crack
\end{abstract}

\section{INTRODUCTION}

As it also happens with the metallic materials, one can assume that the fiber breaking of a composite material starts from small defects, inherent in the material. These defects can have different aspects: fiber micro-cracks, matrix defects, detachments along the interface between fibers and matrix. For a better understanding of the factors affecting the traction behavior of glass-epoxy damaged test bars (with broken fibers), several studies have been carried out, regarding the yielding mechanisms in the proximity of concentrators area. Unlike homogenous materials (metals' behavior can be predicted by computing the stress concentrator while the fibers' behavior by computing the concentration factor at the crack's peak; in the latter case, the main effect is given by the perpendicular propagation of the crack on the main direction of the traction stress.

\section{FIBER BREAKINGS}

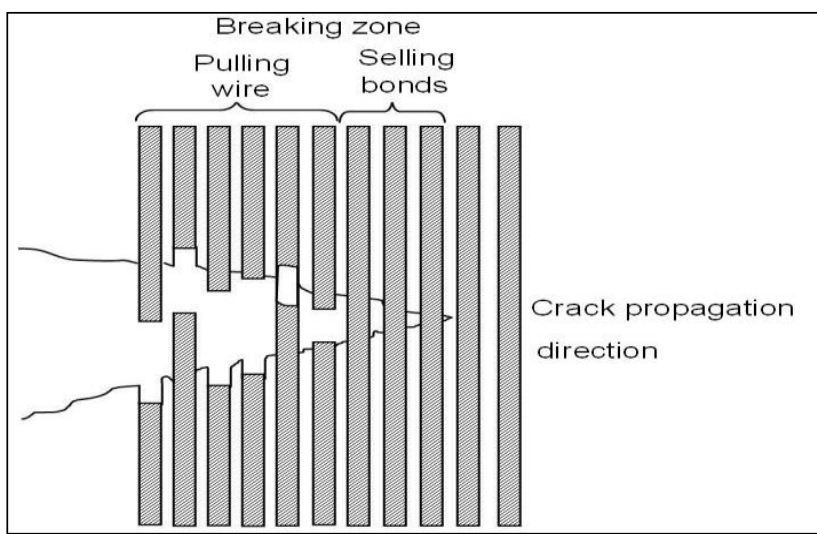

Fig. 1. Crack peak modeling in the fiber reinforced composites

Every time a crack propagates in a normal direction in respect to the fibers' direction, their breaking will lead to a full separation of the layers. The frail fibers, such as graphite fibers, have low breaking strength, which leads to a low energy absorption capacity. The composite's energy per unit area necessary for the fiber fracture under tensile stress is given by the relation

$$
U_{1}=\frac{V_{f} \sigma_{f u}^{2} \ell}{6 E_{f}}
$$

where $V_{f}$ represents the low volume percentage of reinforcement, $\sigma_{f u}$ - the fiber break strength, $E_{f}$ - the fiber longitudinal elasticity modulus and $l$ - the fibers' length. In this respect, Beaumont proposed a similar expression (by replacing the fibers' length with their critical length) in order to characterize the energy released during the fiber breaking process. It was noticed that the number of broken fibers has an insignificant influence on the total impact energy (Belingardi \& Vadori, 2002).

The matrices made of thermo-rigid resins, such as the epoxy or polyester ones, are frail and could undergo a small deformation before breaking. Unlike these, metallic matrices can undergo deep plastic deformations before breaking. The material's matrix cracking and deformation phenomenon occurs through energy absorption. The mechanical work performed during the matrix deformation is proportional to the mechanical work performed during the breaking deformation related to the volume unit $U_{1 m}$ multiplied by the volume of the deformed matrix per unit area of the crack's surface. Taking into account Cooper and Kelly's equation for the volume of the matrix destroyed by cracking, the necessary energy for the matrix fracture per unit area is given by the relation:

$$
U_{1}=\frac{\left(1-V_{f}\right)^{2}}{V_{f}} \frac{\sigma_{m u} d}{4 \tau} U_{1 m}
$$

where $\sigma_{m u}$ is the tensile breaking strength of the matrix, $d$ - the fibers diameter and $\tau$ - shear stress along the interface between fiber and matrix.

During the breaking process, the fibers can detach from the matrix when cracks occur along them. In this process, the secondary or chemical bonds between the fibers and the matrix are destroyed. This kind of crack occurs when the fibers are strong, while the interface between the matrix and fibers is weak. If the breaking of the bonds occurs on a larger surface, a significant increase of the breaking energy can be obtained. An increase of the impact energy can be noticed when the strength of the fiber-matrix interface diminishes. It was shown that the energy produced by the detachment of the fibers can't be matched by the elastic energy stored in the fibers after the detachment (Khondker et al., 2005).

Fiber tearing occurs when in a tenacious matrix discontinuous or fragile fibers are included. Fiber fracture in their weakest section doesn't necessarily occur in the material's breaking plan. The matrix tension concentration determined by the fiber breaking is favored by the yielding of the matrix, thus preventing the matrix cracking phenomenon, which can accompany the phenomenon of fiber breaking in different points. An original analysis was carried out, taking into account the fact that the dissipated energy during the tearing of the discontinuous fibers, with the length $l_{c}$ can be used to approximate the tearing energy of the continuous fibers within 
the composite material, with an equal distribution of the fibers' strengths, assuming that the average value of the torn fibers' length equals $l_{c} / 4$. In other words, the tearing energy per unit of the surface is given by the relation:

$$
U_{1}=\frac{V_{f} \sigma_{f u} l_{c}}{12}
$$

where $V_{f}$ is the fiber volume fraction (volume percentage of the reinforcement).

The tearing of the fibers is usually accompanied by a major matrix deformation, which doesn't occur during the fiber detachment phenomenon. The detachment of the fibers from the matrix, as well as their tearing, is similar phenomenon which can occur in a composite structure, because in both cases the deterioration takes place at the interface (Luo et al., 1999).

A crack propagated along a lamina can be stopped as soon as its peak touches the adjacent fibers. This process is similar to the one related to the arrest of the matrix crack at the interface between fibers and matrix. Because of the high shear stress, the peak of the crack can split, going in a privileged direction parallel to the lamina plan. These types of cracks are also called cracks determined by the structure's delamination and every time they occur, they generate the absorption of a high quantity of breaking strain energy. They frequently occur when the multi-layered material is subject to a bending stress, as it is the case with the impact tests carried out on Charpy-Izod devices.

We may assert that the influence of the material's features under tensile stress and the features related to the impact phenomenon can be considered as being mutually opposing. For instance, a decreased inter-phase strength, which affects the material's tensile and shear strength can lead to an increase in the impact strength. Composite materials made of fibers with a high value of longitudinal elasticity modulus absorb less energy and are much more fragile than fiberglass composite materials with a lower elasticity modulus. Clearly, a compromise can be reached with respect to the interdependence of fiberglass and high elasticity modulus fibers, by combining them in the same composite material.

The impact strength of high elasticity modulus fiber composites generally proved to be rather low as compared to regular steels and aluminum alloys or fiberglass reinforced composites.

An effective method to improve the impact features of graphite fibers reinforced composite materials is to add a small percentage of fibers with high strength (with low elasticity modulus). This method leads to higher performances under impact. In this respect, fiberglass is frequently successfully used. Adding fiberglass, besides from improving the composites' impact performances, also diminishes costs, because they have a lower cost as compared to carbon fibers. The embedment in the matrix of two or more fibers from different materials is called hybridization.

Beaumont and others define an adimensional parameter called ductility index used to establish the impact performances of different materials with similar geometries. The ductility index is defined as the ratio between the propagation energy and the impact fracture initiation energy.

When a composite structure is stressed under impact, the type of deterioration which occurs depends on the incidental energy, material's properties and the test bar's geometry. As a result of a simple calculus, one could determine the energy necessary for the delamination, bending fracture, respectively penetration of the bullet, by using the following relations:

$$
\frac{2}{9} \frac{\tau^{2}}{E} \frac{w l^{3}}{h}, \quad \frac{1}{18} \frac{\sigma^{2}}{E} w l h, \quad \pi \gamma t d
$$

where: $\tau$ and $\sigma[\mathrm{MPa}]$ represent the inter-laminar shear strength, respectively the bending strength; $\gamma[\mathrm{J} / \mathrm{mm}]$ is the thickness fracture energy; $d, w, l, h[\mathrm{~mm}]$ represent the bullet's diameter, respectively width, length and thickness of the bent test bar; $E[M P a]$ is the Young modulus.

Unlike the first two situations, when delamination and bending fracture of the test bar depend on the relative values of $\tau$, respectively $\sigma$ and on the $l / h$ ratio, in the latter case, the bullet will penetrate or not, according to the incidental energy and the bullet's dimensions. The higher the bullet's launching speed, the most likely the penetration. As a general rule, the impact determined by the bullet comes either as a delamination of the composite material, or as fiber bending fracture. (Agarwal et al., 1990)

\begin{tabular}{|l|c|}
\hline \multicolumn{1}{|c|}{ Material } & $\begin{array}{c}\text { Impact energy } \\
{\left[\mathbf{k J / \mathbf { m } ^ { 2 } ]}\right.}\end{array}$ \\
\hline Modmor II, graphyte-epoxy $\left(V_{f}=55 \%\right)$ & 114 \\
\hline Kevlar-epoxy $\left(V_{f}=65 \%\right)$ & 694 \\
\hline S Glass - epoxy $\left(V_{f}=72 \%\right)$ & 694 \\
\hline Nomex-Nylon-epoxy $\left(V_{f}=70 \%\right)$ & 116 \\
\hline Boron-epoxy $\left(V_{f}=60 \%\right)$ & 78 \\
\hline Steel Alloy $\left(\sigma_{r}=700 \rightarrow 1100 \mathrm{MPa}\right)$ & 593 \\
\hline Steel Alloy (Rockwell hardness $=43-46)$ & 214 \\
\hline Refired stainless steel & 509 \\
\hline Aluminum-based alloys & 84 \\
\hline $\begin{array}{l}\text { Aluminum based alloys (solution treated } \\
\text { and precipitation hardened) }\end{array}$ & 153 \\
\hline
\end{tabular}

Tab. 1. Results obtained during Charpy impact test

\section{CONCLUSIONS}

In the case of composite materials, the cracks can propagate in any direction and in any plan in relation to the main stress direction, according to the local tensions as well as to the material's features. Some of these cracks can damage the material's behavior by decreasing its strength. When a composite structure is stressed on impact, the type of the occurring damage depends on the incidental energy, the features of the material and the test bar's geometry. A simple calculus can determine the necessary energy for: delamination, bending fracture, bullet penetration. Studies on notched test bars showed that on traction stress their behavior depends on the yielding mechanisms in the proximity of the notch. the broken fibers decrease the traction strength, while the delaminations among adjacent layers decrease the compression strength.

\section{REFERENCES}

Agarwal, B.D.; Broutman, L.J. \& Chandrashekhara, K. Analysis and Performance of Fiber Composites, Wiley, ISBN 0-471-26891-7, New York, 1990

Avila, A.F.; Marcelo, I.S. \& Almir, S.N. - A study on nanostructured laminated plates behavior under low-velocity impact loadings, International Journal of Impact Engineering, Volume 34, Issue 1, 2007, London, Pages 28-41

Belingardi, G. \& Vadori, R. - Low velocity impact tests of laminate glass-fiber-epoxy matrix composite material plates, International Journal of Impact Engineering, Volume 27, 2002, London, Pages 213-229

Khondker, O.A.; Leong, K.H.; Herszberg, I. \& Hamada, H. Impact and compression-after-impact performance of weftknitted glass textile composites, Composites Part A: Applied Science and Manufacturing, Volume 36, Issue 5, 2005, Pages 638-648

Luo, R.K.; Green, E.R. \& Morrison, C.J. - Impact Damage Analysis of Composite Plates, International Journal of Impact Engineering, Volume 22, 1999, London, Pages 435447 